\title{
Salvianolic Acids Attenuate Rat Hippocampal Injury after Acute CO Poisoning by Improving Blood Flow Properties
}

\author{
Li Guan, ${ }^{1}$ Yan-Lin Zhang, ${ }^{1}$ Zong-Yang Li, ${ }^{1}$ Ming-Xia Zhu, \\ Wei-Juan Yao, ${ }^{2}$ and Jin-Yuan Zhao ${ }^{1}$ \\ ${ }^{1}$ Research Center of Occupational Medicine, Peking University Third Hospital, 49 North Garden Road, Haidian District, \\ Beijing 100191, China \\ ${ }^{2}$ Hemorheology Center, School of Basic Medical Sciences, Peking University Health Science Center, 38 Xue Yuan Road, \\ Haidian District, Beijing 100191, China
}

Correspondence should be addressed to Wei-Juan Yao; weijuanyao@bjmu.edu.cn and Jin-Yuan Zhao; zhaojinyuan.bj@gmail.com

Received 11 August 2014; Accepted 3 November 2014

Academic Editor: Pedro Cabrales

Copyright (c) $2015 \mathrm{Li}$ Guan et al. This is an open access article distributed under the Creative Commons Attribution License, which permits unrestricted use, distribution, and reproduction in any medium, provided the original work is properly cited.

Carbon monoxide (CO) poisoning causes the major injury and death due to poisoning worldwide. The most severe damage via $\mathrm{CO}$ poisoning is brain injury and mortality. Delayed encephalopathy after acute CO poisoning (DEACMP) occurs in forty percent of the survivors of acute CO exposure. But the pathological cause for DEACMP is not well understood. And the corresponding therapy is not well developed. In order to investigate the effects of salvianolic acid (SA) on brain injury caused by CO exposure from the view point of hemorheology, we employed a rat model and studied the dynamic of blood changes in the hemorheological and coagulative properties over acute CO exposure. Compared with the groups of CO and $20 \%$ mannitol + CO treatments, the severe hippocampal injury caused by acute CO exposure was prevented by SA treatment. These protective effects were associated with the retaining level of hematocrit (Hct), plasma viscosity, fibrinogen, whole blood viscosities and malondialdehyde (MDA) levels in red blood cells (RBCs). These results indicated that SA treatment could significantly improve the deformation of erythrocytes and prevent the damage caused by $\mathrm{CO}$ poisoning. Meanwhile, hemorheological indexes are good indicators for monitoring the pathological dynamic after acute CO poisoning.

\section{Introduction}

Carbon monoxide (CO) intoxication is the most common type of poisoning worldwide [1]. CO is a colorless, nonirritating, odorless, and tasteless gas. Despite received various forms of treatments, forty percent of survivors develop delayed encephalopathy after a lucid interval from a few weeks up to months from the acute poisoning. The symptom includes inappropriate euphoria, impaired judgment, poor concentration, memory loss, cognitive and personality changes, psychosis, and Parkinsonism symptoms. A cohort analysis of 256 patients with carbon monoxide poisoning suggests that the severity of the initial symptoms is not correlated to the delayed-onset effects [2]. In addition, neuronal apoptosis, necrosis, and oxidative stress occur within short period (hours or days) after CO poisoning [3-6]. Prevention of these pathologic processes can reduce brain damage and improve the functional recovery and survival rate $[7,8]$. Unfortunately, the etiology of delayed encephalopathy after acute $\mathrm{CO}$ poisoning (DEACMP) remains unclear.

DEACMP happens more often in the elders with cardiovascular or cerebrovascular diseases [9]. Furthermore, the regions with poor vasculatures in brain such as white matter and basal ganglia are also very sensitive to hypoxic damage $[10,11]$. This association of vasculature properties and DEACMP indicates that the disturbance of cerebral circulation may have a high impact on the induction of delayed cerebral injury after $\mathrm{CO}$ exposure.

Hemorheological parameters are the most important vasculature properties. Hemorheology is an important subject that studies the flow properties of blood circulation and its elements (plasma and formed elements, including red 
blood cells, white blood cells, and platelets). Whole blood viscosity and erythrocyte aggregation and deformation are key hemorheological parameters [12]. Collective evidences indicate that the flow properties of blood are among the main determinants of proper tissue perfusion and the alterations, which play significant roles in many disease processes [1317]. It has been shown that hemorheological derangement (i.e., whole blood, plasma, and serum hyperviscosity, reduced erythrocyte deformability, increased red cell aggregation, and hyperfibrinogenemia) can potentially impair microand macrovascular blood flow in the brain of Alzheimer's disease subjects [18]. The hemorheologic changes are also important factors in determining the clinical outcomes for the patients with coronary or cerebral arterial diseases [1921]. We hypothesize that the development of DEACMP may be associated with the deterioration of hemorheological parameters after $\mathrm{CO}$ exposure. Therefore, in the present study, we investigated the relationship between DEACMP and hemorheologic dynamic changes.

Salvianolic acid (SA) is commonly used in clinical trials to decrease blood viscosity. SA is a natural product isolated from the Chinese traditional medicine, Salvia miltiorrhiza. Besides improving the blood circulation [22], SA is also a strong antioxidant with the activity of inhibiting lipid peroxidation, scavenging hydroxyl radical, preventing platelet aggregation, and so forth $[23,24]$.

In this study, we employed a rat model to investigate the dynamic of hemorheological changes at different time after acute $\mathrm{CO}$ poisoning. We found that the treatment of SA significantly improved the hemorheological properties and, more importantly, prevented the neuronal damage. This indicates that the improvement of blood flow properties could indeed relieve the neuronal damage in $\mathrm{CO}$ poisoning. This study provides the new insight of $\mathrm{CO}$ toxicology to central nervous system effects in perspectives of hemorheology and the administration of SA to prevent of DEACMP.

\section{Materials and Methods}

2.1. Animals. Male Sprague-Dawley rats (300-350 g) were obtained from the Peking University Animal Breeding Unit. All animals were raised under the same laboratory conditions of temperature $\left(25^{\circ} \mathrm{C}\right)$ and lighting (12/12 h light/dark cycle) and were given free access to standard laboratory chow and tap water. All rats were allowed to acclimatize for 1 week prior to experimentation. All experimental procedures involving animals were approved by the Ethical Animal Committee of Peking University, Beijing, China.

2.2. Reagents. Carbon monoxide was purchased from Beijing Beiyang Special Institute Co. (China); SA was supplied by the Modern Research Center for Traditional Chinese Medicine of Peking University. PVP- (polyvinyl pyrrolidone-) K30 was provided by Shanghai Chemical Instrument Co. MDA assay kit was purchased from Nanjing Jiancheng Agent Co. All other chemicals were of analytical grade from commercial suppliers.
2.3. Treatments. All substances were dissolved in pyrogenfree saline unless indicated otherwise. Experimental animal groups were as follows. (1) CO exposure group $(n=15)$ : CO poisoning (more than $70 \%$ carboxyhemoglobin in the blood) was induced in rats by exposure to air supplemented with $3000 \mathrm{ppm}$ CO introduced into the chamber at a flow rate of $8 \mathrm{~L} / \mathrm{min}$ for $40 \mathrm{~min}[25,26]$. (2) Control group $(n=25)$ : rats were treated with air by the same method. (3) Salvianolic acids (SA) + CO exposure $(n=25):$ SA $(6 \mathrm{mg} / \mathrm{kg}$ body weight /day) was administered to rats every day via the tail vein after CO poisoning [27]. (4) $20 \%$ mannitol + CO exposure $(n=$ 25): $20 \%$ mannitol (10 mL/kg body weight) was administered every day via the tail vein after CO treatment [28]. The blood samples were collected from eye socket at $1 \mathrm{~h}, 1-5$ days after CO exposure for detection of various hemorheological parameters. Rats were sacrificed at day 5 and their brains were removed for histochemical stains. The rats had free access to drinking water during the experimental period.

2.4. Histochemical Staining and Counting of Necrotic Neurons. For analysis of hippocampal neuronal survival, rats that survived for 5 days were deeply anesthetized with halothane, perfused through the heart with approximately $200 \mathrm{~mL}$ phosphate-buffered saline ( $0.05 \mathrm{M}$ sodium phosphate $\mathrm{pH} 7.2,0.1 \mathrm{M} \mathrm{NaCl}$ ), and perfusion was fixed with $4 \%$ paraformaldehyde. Brains were removed and fixed in $4 \%$ paraformaldehyde before paraffin embedding and dissection. Serial sections $(5 \mu \mathrm{m})$ were cut (corresponding to bregma $-3.3 \mathrm{~cm}$ according to the atlas of Paxinos and Watson 11) and stained with hematoxylin and eosin ( $\mathrm{H} \& \mathrm{E})$. Viable pyramidal neurons were counted in the CA1 region of the hippocampus under a high-power field $(\times 400)$. Necrotic neurons were recognized by their pyknotic or karyorrhectic nuclei lacking clear nucleoli, whereas viable neurons were defined as those cells showing distinct nuclear and nucleolar structures. The number of normal pyramidal neurons per high-power field was counted by an observer blinded to the experimental protocol.

2.5. Hematological Analysis and Measurement of Plasma, Whole Blood Viscosities, and Fibrinogen Concentration. Heparinized blood samples were drawn from eye socket of rats 1 h, 1 5 days after CO exposure. Twenty microliters of fresh blood was applied to a full-automated hematological analyzer (Sysmex KX-21N, Japan) for determination of hematocrit (Hct). Plasma was separated by centrifuging blood at $150 \mathrm{~g}$. The plasma viscosity and whole blood viscosity at different shear rates $\left(200,50\right.$, and $\left.5 \mathrm{~s}^{-1}\right)$ were measured with an automatic viscometer (LG-R-80B, Steellex Co., Beijing, China). Fibrinogen concentration was measured using coagulant analyzer (RABRE, Steellex Co., Beijing, China).

2.6. Measurement of Deformation Index and Aggregation Index of Red Blood Cells (RBCs). Red blood cells (RBCs) were suspended in $15 \%$ PVP buffer $(w / V, M W=30 \mathrm{kDa}$, $61 \mathrm{mM} \mathrm{NaCl}, 0.8 \mathrm{mM} \mathrm{Na}_{2} \mathrm{HPO}_{4}$, pH 7.4, $290 \mathrm{mOsm} / \mathrm{kg}$, and viscosity $15 \mathrm{mPas}$ ) and adjusted to the concentration of $2 \times$ $10^{7} / \mathrm{mL}$. Bovine serum albumin (BSA) of $40 \mathrm{mg} / \mathrm{mL}$ was 


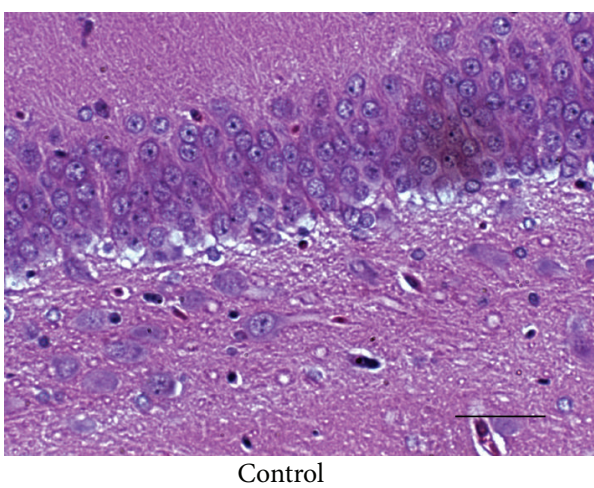

(a)

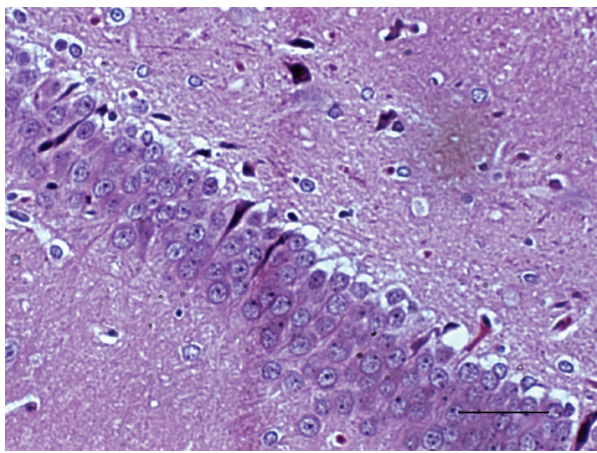

$\mathrm{SA}+\mathrm{CO}$ exposure

(c)

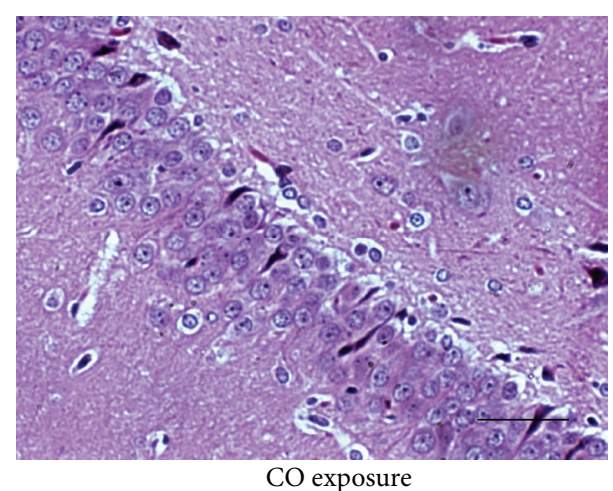

(b)

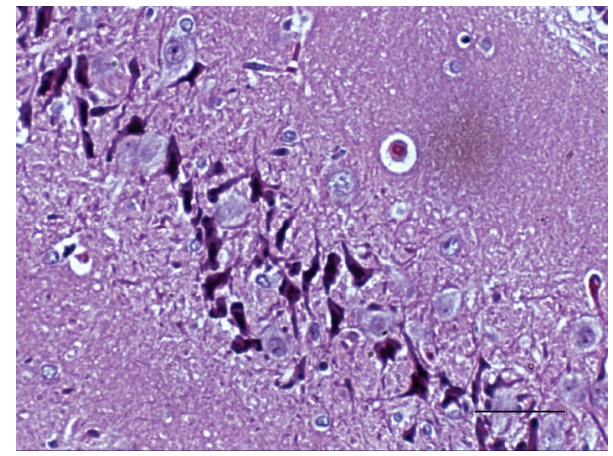

$20 \%$ mannitol $+\mathrm{CO}$ exposure

(d)

FIGURE 1: Neuronal survival and necrosis in the CA1 region of CO-exposed rates. The panels show representative H\&E stained sections from control group (a), the CO exposure group (b), the SA + CO group (c), and the $20 \%$ mannitol + CO group (d). (a) The control rats, with rare neuronal death occurring in the $\mathrm{CA} 1$ region; (b) CO-exposed rats, with massive neuronal degeneration, apoptosis, or necrosis, and neuronal density decreased, as arrows indicated; (c) SA + CO group rats: neuronal density and cell morphology were well preserved; only mild degeneration of neuron could be observed occasionally; (d) 20\% mannitol + CO exposure rats: severe damage of neuron was observed in CA1 region which was more serious than CO-exposed alone rats, as arrows indicated. All images were captured at 400x magnification; calibration bar, $100 \mu \mathrm{m}$.

added into the buffer to keep the morphology of RBCs. The deformation indices (DI) at shear rates of $50-1000 \mathrm{~s}^{-1}$ were measured with a traditional ektacytometer (Model LBY-BX2, Precil Co., Beijing, China) [29]. Five hundred microliters of blood was used for RBC aggregation measurement with a photometric aggregometer (Steellex Co., Beijing, China) interfaced to a computer. The cells were sheared at $1000 \mathrm{~s}^{-1}$ for $5 \mathrm{~s}$. After the shearing, the change of light transmission through RBC suspension was monitored and an aggregation rate was calculated.

2.7. Estimation of Lipid Peroxidation of RBCs. MDA determination is based on spectrophotometric or spectrofluorimetric measurement of the condensation product formed from MDA and 2-thiobarbituric acid (TBA) by measuring the absorbance at $532 \mathrm{nM}$ after reaction.

2.8. Statistical Analyses. The data is expressed as mean \pm SD. Statistical analyses were performed in SPSS13.0 software. The differences between groups were analyzed by ANOVA and considered to be statistically significant when $P<0.05$.

\section{Results}

3.1. The Pathological Changes in Hippocampus. Brain tissue sections from each group were H\&E stained and examined under the light microscope on day 5 . In $\mathrm{CO}$ group and $20 \%$ mannitol + CO group, the hippocampus displayed an abnormal histology, with marked changes in the thickness of the pyramidal cell layers (Figure 1). The numbers of neurons in the CA1 subfield were evaluated by counting the viable neurons in each field. The neuron densities reduced by $29 \%$ and $63 \%$, respectively, in animals treated with $\mathrm{CO}$ or $\mathrm{CO}$ $+20 \%$ mannitol (Figure 1 and Table 1). In SA + CO group, however, the number of surviving neurons ( $72 \pm 9$ per field) was significantly higher than $\mathrm{CO}$ group $(58 \pm 10$ per field). The difference between $\mathrm{SA}+\mathrm{CO}$ group and the control group was not statistically significant $(P>0.05)$.

3.2. Characteristics of Hematological Parameters in COPoisoned Rats. The hematocrit (Hct) changes after $\mathrm{CO}$ exposure were presented in Figure 2. In CO group, Hct slightly dropped at $1 \mathrm{~h}$ and started to increase from day $1(P<0.05$ 
TABLE 1: The numbers of damaged neurons in the hippocampal CAl region of rats in different groups.

\begin{tabular}{|c|c|c|c|c|}
\hline \multirow{2}{*}{ Hippocampal region } & \multicolumn{4}{|c|}{ Group } \\
\hline & Control & CO exposure & $\mathrm{SA}+\mathrm{CO}$ exposure & $20 \%$ mannitol + CO exposure \\
\hline CA1 subfield & $82 \pm 7$ & $58 \pm 10^{*}$ & $72 \pm 9^{\#}$ & $30 \pm 4^{* / \#}$ \\
\hline
\end{tabular}

Note. Data are means $\pm \mathrm{SEM} ;{ }^{*} P<0.01$ versus control; ${ }^{\#} P<0.01$ versus CO exposure.

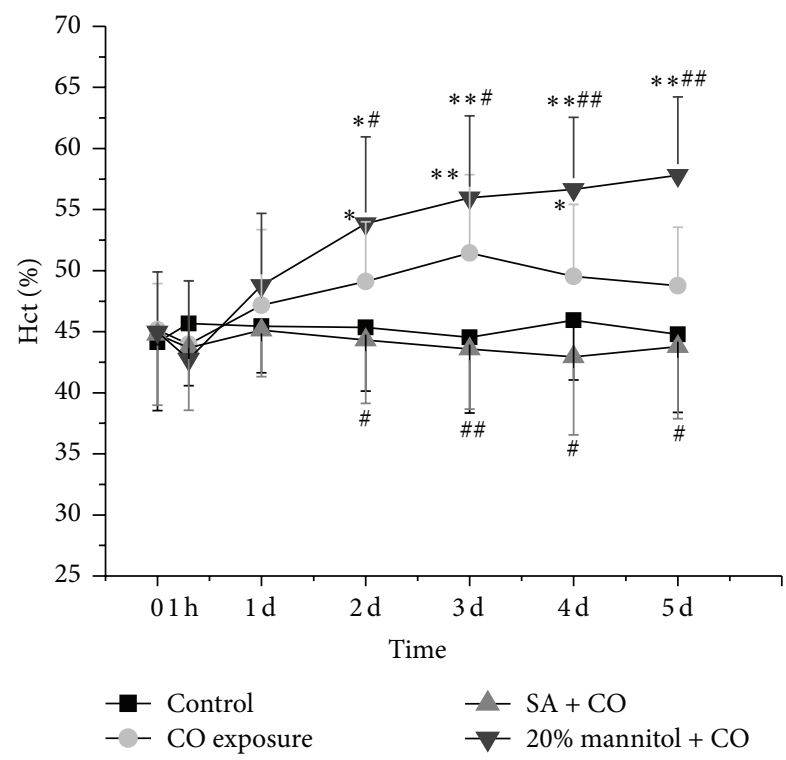

FIgURE 2: Comparison of Hct among the CO exposure group, the SA $+\mathrm{CO}$ group, the $20 \%$ mannitol + CO group, and the control group. ${ }^{*} P<0.05$ and ${ }^{* *} P<0.01$ versus control group. ${ }^{*} P<0.05$ and ${ }^{\# \#} P<0.01$ versus $\mathrm{CO}$ exposure group.

compared to control) and reached the highest level on day 3 $(P<0.01)$. There was a little drop afterwards but it was still higher than control $(P<0.05)$. In $20 \%$ mannitol + CO group, Hct was significantly elevated compared to $\mathrm{CO}$ group from day $1(P<0.01)$. In SA + CO group, the elevation of Hct was greatly prevented by SA infusion.

The plasma viscosities and fibrinogen concentrations in different groups were detected (Figures 3(a) and 3(b)). The plasma viscosities and fibrinogen concentrations rose at 1 hour and kept increasing to the highest level on day $3(P<$ 0.05 (Figure 3(a)); $P<0.01$ (Figure 3(b))) in both $\mathrm{CO}$ and $20 \%$ mannitol + CO groups. On day 5, both of them still remained higher than control. No significant difference in Hct, plasma viscosity, and fibrinogen concentration between $\mathrm{SA}+\mathrm{CO}$ group and control was observed on days 1-5.

3.3. The Changes in Whole Blood Viscosity. The whole blood viscosities at high $\left(200 s^{-1}\right)$, medium $\left(50 s^{-1}\right)$, and low $\left(5 s^{-1}\right)$ shear rate showed similar pattern (Figures 4(a), 4(b), and $4(c))$. They all decreased significantly $(P<0.01)$ at 1 hour after $\mathrm{CO}$ exposure. But, 1 day later, they began to increase and recovered to the control level on day 5 in CO group. While in $20 \%$ mannitol + CO group the whole blood viscosities kept high level until day $5(P<0.01)$, with $\mathrm{SA}+\mathrm{CO}$ treatment, the whole blood viscosities recovered to control level 1 day after CO exposure and remained at a similar level to that of control group.

3.4. Changes of the Deformability and Aggregation of Erythrocytes. The deformation index (DI) and aggregation index were measured and the data were shown in Figures 5(a) and 5(b). DI decreased at $1000 \mathrm{~s}^{-1}$ at 1 hour after CO exposure $(P<0.01)$. It slightly increased on day 1 and gradually reached the control level on day 5 in CO group. In SA + CO group, DI obviously increased on day 1 when compared with $\mathrm{CO}$ and $20 \%$ mannitol $+\mathrm{CO}$ groups $(P<0.01)$. There was no significant difference between control group and SA + CO group from day 1 to day 5 .

The aggregation index of CO group dropped significantly at 1 hour after $\mathrm{CO}$ exposure and started to recover on day 1 . Finally it approached the normal level by day 5 . In SA + CO group, the increase in aggregation index was more significant than the CO group from day $1(P<0.05)$. But, in $20 \%$ mannitol + CO group, the aggregation index began to greatly increase from $0.55 \pm 0.13$ to $1.35 \pm 0.12$ on day 1 and remained high till day 5 .

3.5. Changes of MDA in Erythrocytes. The MDA levels in erythrocytes were measured and shown in Figure 6. The MDA levels kept increasing along with the treatment in $\mathrm{CO}$ group $(P<0.01)$, while in SA + CO group MDA levels were lower than those in CO and 20\% mannitol + CO groups from day $1(P<0.05)$, despite that they were still slightly higher than those in the control group.

\section{Discussion}

In the present study, we found that $\mathrm{CO}$ poisoning caused the deterioration of hemorheological properties and severe neuronal damage in rats. The treatment of SA significantly improved the blood flow and coagulant properties and, more importantly, prevented the neuronal damage.

Normal deformability of RBCs, also known as erythrocyte deformability, is an important property for the transportation of oxygen in blood as well as the normal perfusion of microcirculation [30,31]. Decreased deformability of RBCs may lead to a reduction of life span of RBCs as well [32]. Cicco and Pirrelli reported that as low as $10 \%$ decrease in erythrocyte deformability in hypertensive patients might lead to a significant impairment in both cellular and tissue oxygen delivery [33]. Our study showed that DI decreased after CO exposure, reflecting a definite change in deformability of RBCs. Erythrocyte deformability in CO and 20\% mannitol + CO groups decreased about $28 \%$ and $53 \%$, respectively. These indicated that there were dysfunctions in cellular oxygen 


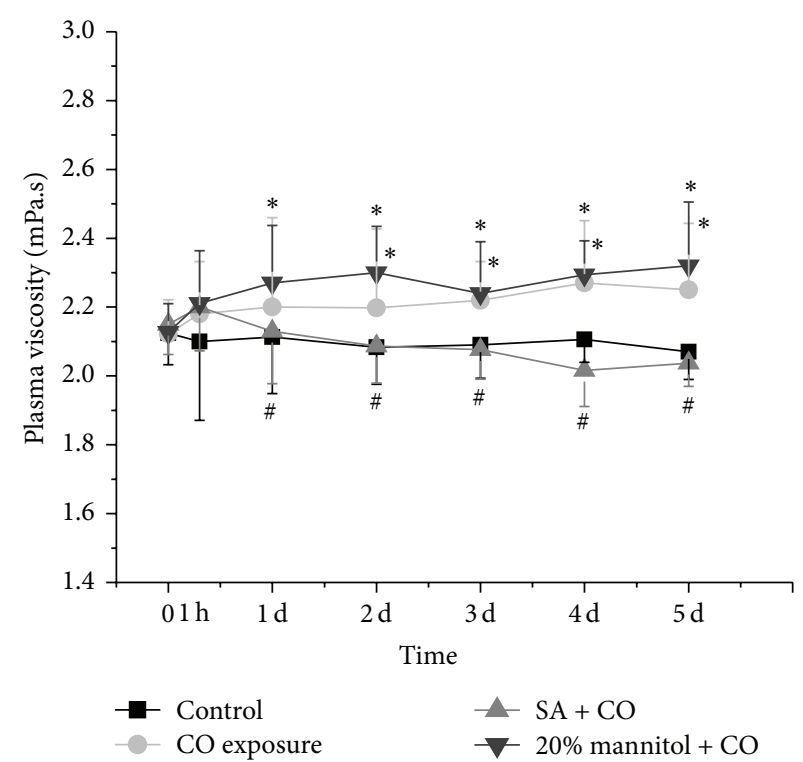

(a)

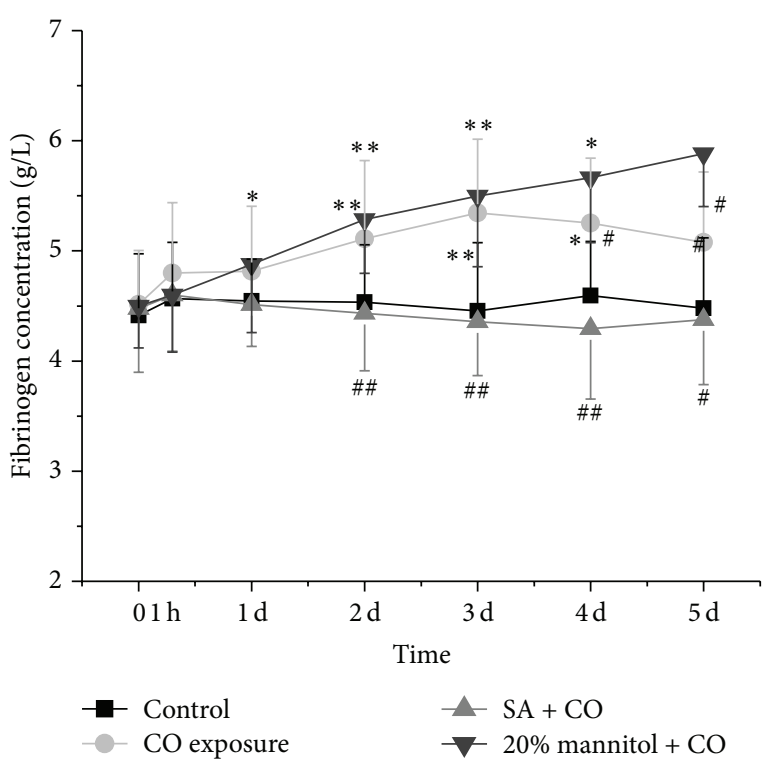

(b)

FIGURE 3: Comparison of plasma viscosity and fibrinogen concentration among the CO exposure group, the SA + CO group, the $20 \%$ mannitol + CO group, and the control group. ${ }^{*} P<0.05$ and ${ }^{* *} P<0.01$ versus control group. ${ }^{\#} P<0.05$ and ${ }^{\# \#} P<0.01$ versus CO exposure group.

delivery and tissue oxygen delivery in CO-exposed animals. Furthermore, this change can be prevented by the application of SA. Erythrocyte deformability was improved nearly $20 \%$ in $\mathrm{SA}+\mathrm{CO}$ group, which suggested the therapeutical potential of SA administration for CO poisoning.

Our study also showed MDA level in RBCs, which is one of the most important ending products of lipid peroxidation. MDA presented an abrupt increase at the very beginning of $\mathrm{CO}$ exposure and remained high till the end of the experiment (5 days after $\mathrm{CO}$ exposure). This data suggests there was an enhancement of lipid peroxidation after $\mathrm{CO}$ exposure. The corresponding oxidant stress may decrease the erythrocyte deformability in the early stage. Lipid peroxidation may or may not be the direct reason for the impairment of erythrocyte deformability, because the membrane protein components, the major determinants for RBC mechanical properties, are independent upon lipid peroxidation. Condon et al. found that depletion of white blood cell (WBC) was sufficient to abrogate the ability of trauma hemorrhagic shock (T/HS) lymph to cause abnormal RBC deformability, indicating that RBC injury by T/HS lymph requires WBC [34]. It is likely that the enhanced oxidant stress induced polymorphonuclear neutrophils (PMN) respiratory burst activity. As a result, multiple mediator networks were stimulated, such as complements, kinins, coagulation, and fibrinolysis cascades, along with the release of chemokines, cytokines, soluble receptors, lipid mediators, and numerous enzymes such as elastase, myeloperoxidase, and proteases. The net result could lead to the changes of RBC hemorheology properties and various pathological abnormalities after $\mathrm{CO}$ exposure.

Another important property of RBCs is the ability to aggregate. In large vessels, RBC aggregates flow in the center of the vessels, which create a cell-free layer near the vessel wall, thereby facilitating flow. In smaller vessels, the flowinduced shear stress disperses the aggregates so RBCs can flow through the capillaries [35, 36]. It is known that erythrocytes tend to aggregate with lower velocities of circulating blood, and erythrocyte aggregation may contribute to the margination and adhesion of leukocytes and platelets to the vessel wall [37]. Erythrocyte aggregation is one of the key factors of whole blood viscosity at low shear rates [38]. When $\mathrm{RBC}$ aggregability is enhanced, the aggregates could obstruct capillary blood flow and inhibit tissue oxygenation. Yet, in this study, we found that erythrocyte aggregation decreased rapidly right after $\mathrm{CO}$ exposure (Figure 5(b)) and it returned to the control level on day 4 . The decreased erythrocyte aggregation may contribute to the reduction of whole blood viscosity at low shear rates $1 \mathrm{~h}$ after intoxication but may not be beneficial for leukocyte and platelets adhesion. The reason that caused $\mathrm{RBC}$ aggregation change should be further studied.

SA blocked the CO-induced increase in hippocampal injury and improved the hemorheological parameters, including Hct, plasma viscosity, fibrinogen, and whole blood viscosities. In response to $\mathrm{CO}$ exposure, the whole blood viscosities dipped in a quick fashion and recovered 24 hours later (Figure 4), while in CO and 20\% mannitol + $\mathrm{CO}$ groups the whole blood viscosities not only returned to the normal value but also continued increasing till 5 days after the exposure (Figure 5). CO causes lipid peroxidation by releasing nitric oxide (NO) and oxygen-free radicals from endothelial cell and platelet [39]. Therefore, a compensative and protective reaction to hypoxia in microcirculation perfusion after $\mathrm{CO}$ exposure might happen. There are several interpretations based on this observation. The widely 


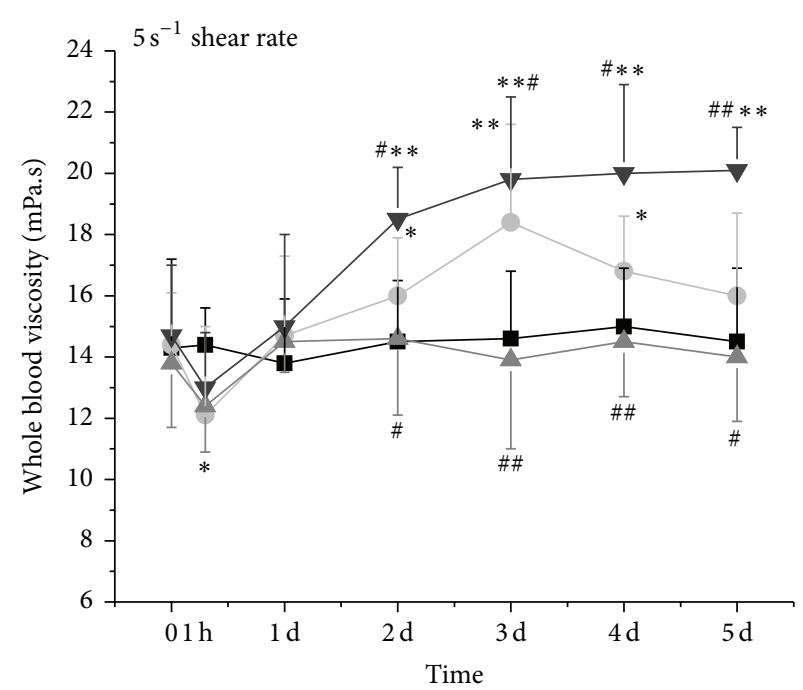

(a)

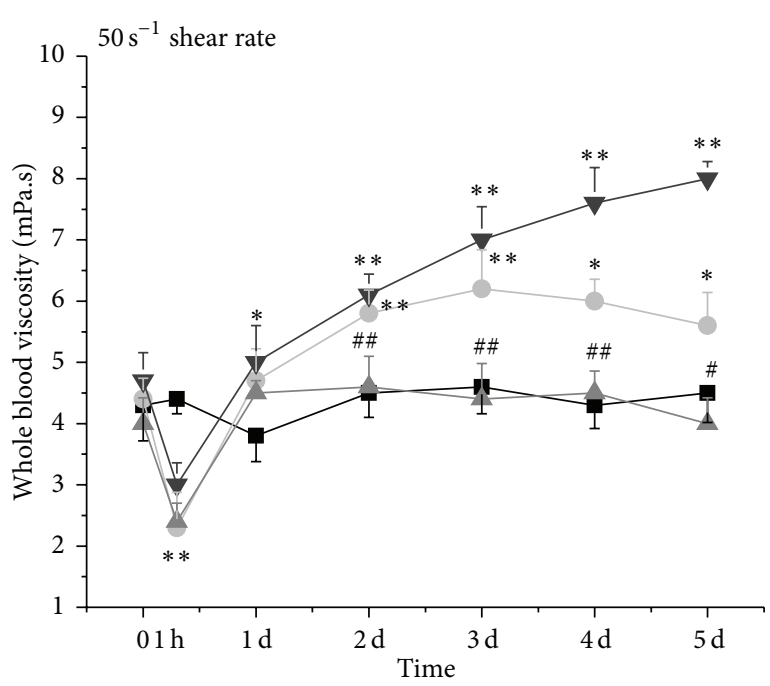

(b)

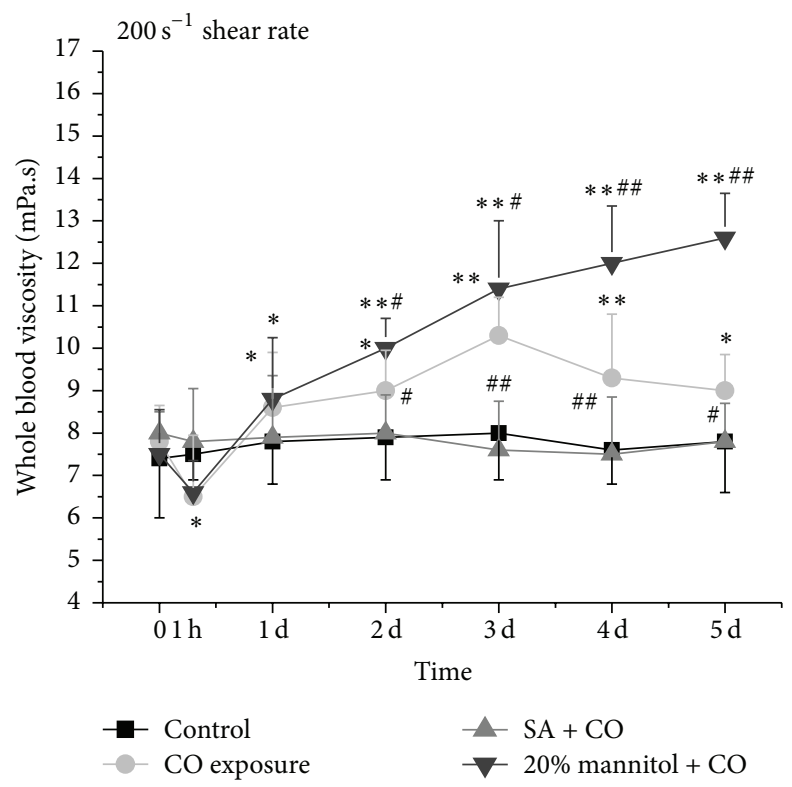

(c)

Figure 4: Comparison of whole blood viscosities at high, medium, and low shear rates among CO exposure group, SA + CO group, 20\% mannitol $+\mathrm{CO}$ group, and control group. ${ }^{*} P<0.05$ and ${ }^{* *} P<0.01$ versus control group. ${ }^{\#} P<0.05$ and ${ }^{\# \#} P<0.01$ versus CO exposure group.

accepted view is that the increase in blood flow may be able to supply enough oxygen so that there is no change in the oxygen consumption. Another interpretation is that the increase in blood flow may cause vasodilation independently based upon oxygen level with dilatory agent (such as NO) and maintain adequate tissue oxygen level with the increased oxygen extraction [40]. But the change in whole blood viscosity at low shear rate occurring on day 1 after $\mathrm{CO}$ exposure could have disturbed the microcirculation in brain, especially in the regions with poor vasculatures, such as white matter, globus pallidus, basal ganglia, and hippocampus. The constant increase in plasma fibrinogen and changes with hematocrit after 2 days may contribute to the increase in whole blood viscosity (Figures 2 and 3 ).

\section{Conclusion}

Our study first reported that there were hemorheological changes in brain after acute $\mathrm{CO}$ exposure. The decrease in the whole blood viscosity as a compensatory reaction at early stage may contribute to accelerating brain blood flow and improving oxygen cerebral supply. But the increase in whole blood viscosity may affect the blood perfusion in heart 


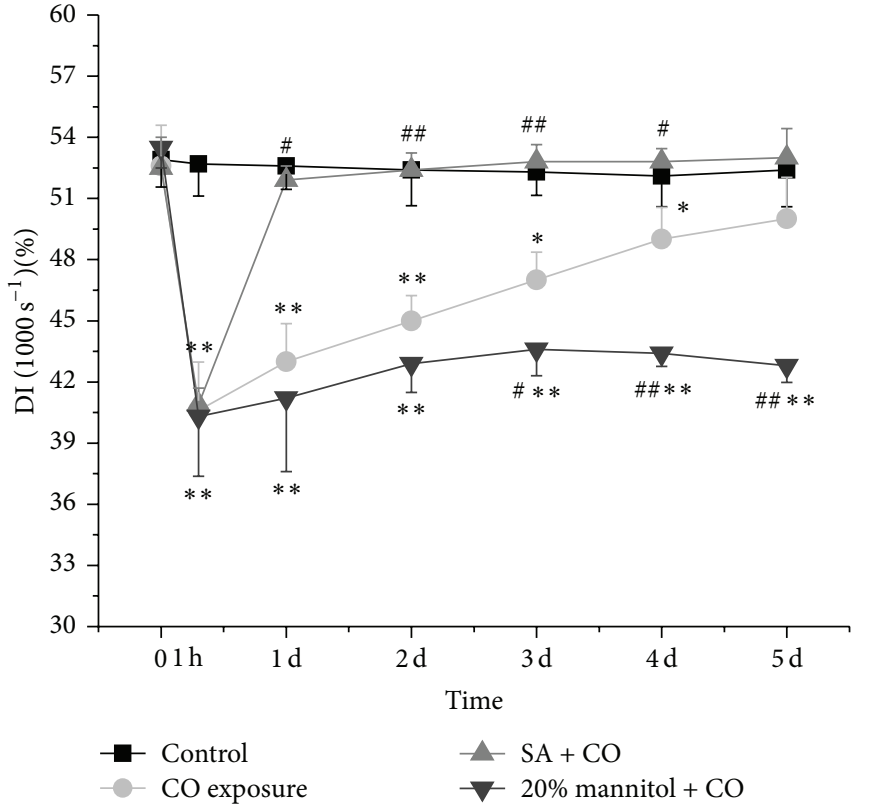

(a)

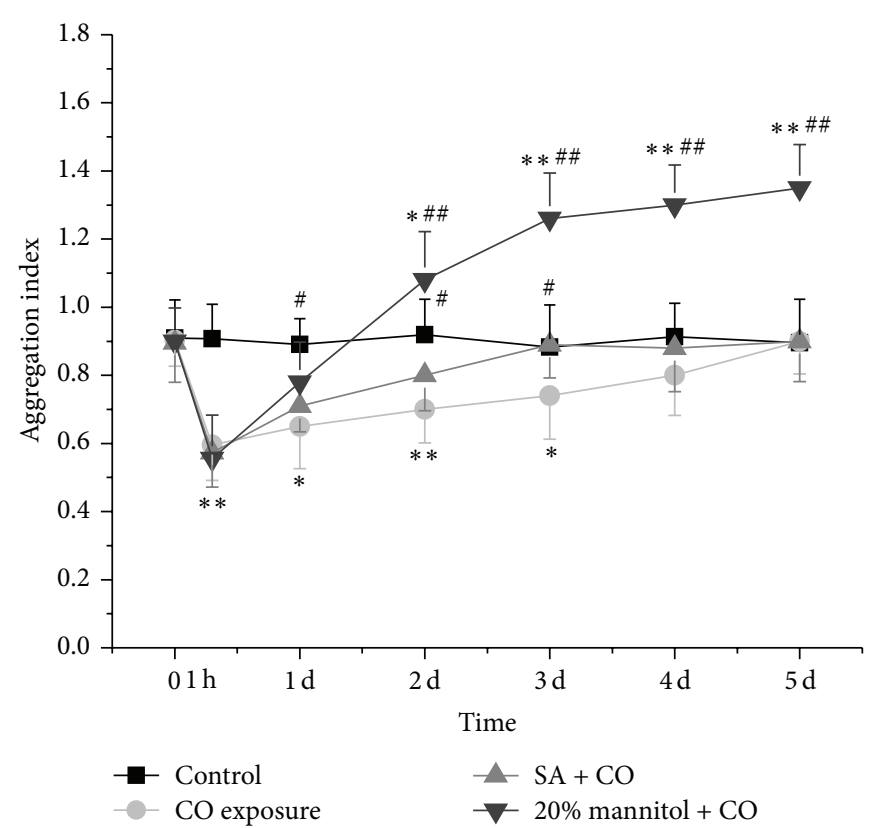

(b)

FIGURE 5: Comparison of deformability and aggregation of erythrocytes among the CO exposure group, the SA + CO group, the $20 \%$ mannitol + CO group, and the control group. ${ }^{*} P<0.05$ and ${ }^{* *} P<0.01$ versus control group. ${ }^{\#} P<0.05$ and ${ }^{\# \#} P<0.01$ versus CO exposure group.

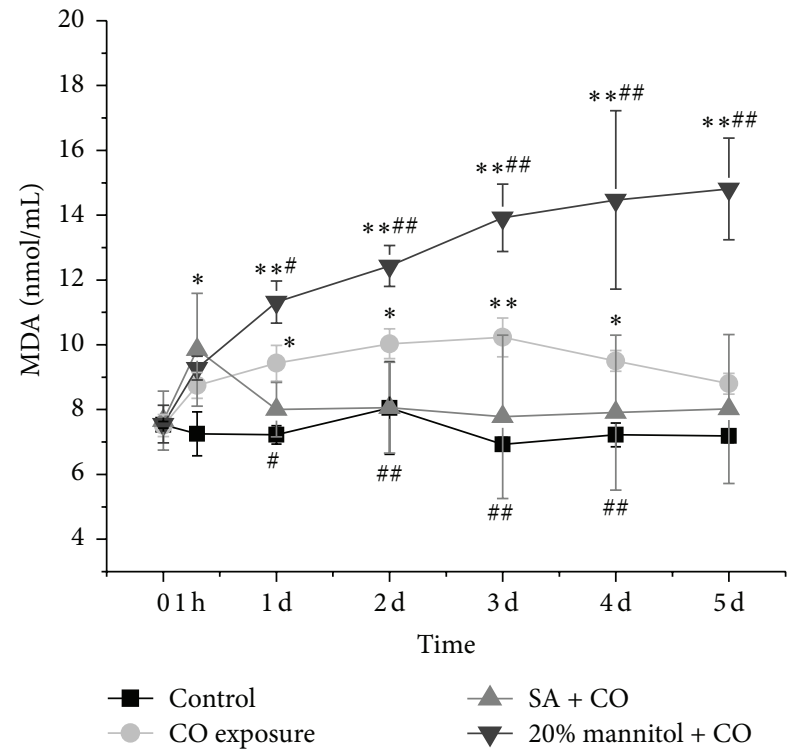

FIGURE 6: Comparison of MDA level among the CO exposure group, the SA + CO group, the $20 \%$ mannitol + CO group, and the control group. ${ }^{*} P<0.05$ and ${ }^{* *} P<0.01$ versus control group. ${ }^{\#} P<0.05$ and ${ }^{\# \#} P<0.01$ versus $C O$ exposure group.

and cerebral microcirculations and even result in ischemia especially in the regions with poor vasculature such as white matter, globus pallidus, basal ganglia, and hippocampus. The study also showed that SA could efficiently clean out the free radicals, well improve the deformability of RBCs after
$\mathrm{CO}$ exposure, and greatly relieve hypoxemia and pathological abnormalities of hippocampus.

\section{Conflict of Interests}

The authors declare that there is no conflict of interests regarding the publication of this paper.

\section{Acknowledgments}

This work was supported by the National Natural Science Foundation of China, Grant no. 81101024, and Beijing Natural Science Foundation, Grant no. 7113175. Thanks are due to the English Language Editing Service at the International Science Editing and Dr. XueYing Guan who works at Section of Molecular Cell and Developmental Biology Center for Computational Biology and Bioinformatics, University of Texas at Austin.

\section{References}

[1] S. Iqbal, J. H. Clower, S. A. Hernandez, S. A. Damon, and F. Y. Yip, "A review of disaster-related carbon monoxide poisoning: surveillance, epidemiology, and opportunities for prevention," American Journal of Public Health, vol. 102, no. 10, pp. 1957-1963, 2012.

[2] C. A. Chambers, R. O. Hopkins, L. K. Weaver, and C. Key, "Cognitive and affective outcomes of more severe compared to less severe carbon monoxide poisoning," Brain Injury, vol. 22, no. 5, pp. 387-395, 2008. 
[3] C. A. Piantadosi, J. Zhang, E. D. Levin, R. J. Folz, and D. E. Schmechel, "Apoptosis and delayed neuronal damage after carbon monoxide poisoning in the rat," Experimental Neurology, vol. 147, no. 1, pp. 103-114, 1997.

[4] R. Song, Z. Zhou, P. K. M. Kim et al., "Carbon monoxide promotes Fas/CD95-induced apoptosis in Jurkat cells," The Journal of Biological Chemistry, vol. 279, no. 43, pp. 44327-44334, 2004.

[5] S. R. Thom, D. Fisher, Y. A. Xu, K. Notarfrancesco, and H. Lschiropoulos, "Adaptive responses and apoptosis in endothelial cells exposed to carbon monoxide," Proceedings of the National Academy of Sciences of the United States of America, vol. 97, no. 3, pp. 1305-1310, 2000.

[6] V. Turcanu, M. Dhouib, J.-L. Gendrault, and P. Poindron, "Carbon monoxide induces murine thymocyte apoptosis by a free radical-mediated mechanism," Cell Biology and Toxicology, vol. 14, no. 1, pp. 47-54, 1998.

[7] L. D. Prockop and R. I. Chichkova, "Carbon monoxide intoxication: an updated review," Journal of the Neurological Sciences, vol. 262, no. 1-2, pp. 122-130, 2007.

[8] L. Guan, T. Wen, Y. Zhang, X. Wang, and J. Zhao, "Induction of heme oxygenase-1 with hemin attenuates hippocampal injury in rats after acute carbon monoxide poisoning," Toxicology, vol. 262, no. 2, pp. 146-152, 2009.

[9] C. R. Henry, D. Satran, B. Lindgren, C. Adkinson, C. I. Nicholson, and T. D. Henry, "Myocardial injury and longterm mortality following moderate to severe carbon monoxide poisoning," The Journal of the American Medical Association, vol. 295, no. 4, pp. 398-402, 2006.

[10] N.-C. Chen, W.-N. Chang, C.-C. Lui et al., "Detection of gray matter damage using brain MRI and SPECT in carbon monoxide intoxication: a comparison study with neuropsychological correlation," Clinical Nuclear Medicine, vol. 38, no. 2, pp. e53e59, 2013.

[11] N.-C. Chen, C.-W. Huang, C.-C. Lui et al., "Diffusion-weighted imaging improves prediction in cognitive outcome and clinical phases in patients with carbon monoxide intoxication," Neuroradiology, vol. 55, no. 1, pp. 107-115, 2013.

[12] O. H. M. Baskurt, M. W. Rampling, and H. J. Meiselman, Handbook of Hemorheology and Hemodynamics, IOS Press, Amsterdam, The Netherlands, 2007.

[13] M. Eugster, K. Häusler, and W. H. Reinhart, "Viscosity measurements on very small capillary blood samples," Clinical Hemorheology and Microcirculation, vol. 36, no. 3, pp. 195-202, 2007.

[14] G. Mchedlishvili, "Hemorheological changes in microcirculation: their mechanism and measurement technique," Indian Journal of Experimental Biology, vol. 45, no. 1, pp. 32-40, 2007.

[15] S. Li, C. Wu, L. Zhu et al., "By improving regional cortical blood flow, attenuating mitochondrial dysfunction and sequential apoptosis galangin acts as a potential neuroprotective agent after acute ischemic stroke," Molecules, vol. 17, no. 11, pp. 1340313423, 2012.

[16] E. Pytel, P. Duchnowicz, P. Jackowska, K. Wojdan, M. KoterMichalak, and M. Broncel, "Disorders of erythrocyte structure and function in hypertensive patients," Medical Science Monitor, vol. 18, no. 8, pp. BR331-BR336, 2012.

[17] J. Yeboah, R. L. McClelland, T. S. Polonsky et al., "Comparison of novel risk markers for improvement in cardiovascular risk assessment in intermediate-risk individuals," Journal of the American Medical Association, vol. 308, no. 8, pp. 788-795, 2012.

[18] S. B. Solerte, G. Ceresini, E. Ferrari, and M. Fioravanti, "Hemorheological changes and overproduction of cytokines from immune cells in mild to moderate dementia of the Alzheimer's type: adverse effects on cerebromicrovascular system," Neurobiology of Aging, vol. 21, no. 2, pp. 271-281, 2000.

[19] H. Kim, Y. I. Cho, D.-H. Lee et al., "Analytical performance evaluation of the scanning capillary tube viscometer for measurement of whole blood viscosity," Clinical Biochemistry, vol. 46, no. 1-2, pp. 139-142, 2013.

[20] I. Ricci, F. Sofi, A. Alessandrello Liotta et al., "Alterations of haemorheological parameters in patients with peripheral arterial disease," Clinical Hemorheology and Microcirculation, vol. 55, no. 2, pp. 271-276, 2013.

[21] L. Mannini, R. Paniccia, E. Cecchi et al., "Reduced erythrocyte deformability and hypercoagulability in idiopathic sudden sensorineural hearing loss," Clinical Hemorheology and Microcirculation, vol. 33, no. 1, pp. 47-55, 2005.

[22] J. Wang, X. Xiong, and B. Feng, "Cardiovascular effects of salvianolic acid B," Evidence-based Complementary and Alternative Medicine, vol. 2013, Article ID 247948, 16 pages, 2013.

[23] C. Y. Wang, F. L. Ma, J. T. Liu, J. W. Tian, and F. H. Fu, "Protective effect of salvianic acid a on acute liver injury induced by carbon tetrachloride in rats," Biological and Pharmaceutical Bulletin, vol. 30, no. 1, pp. 44-47, 2007.

[24] J. Hou, J. Tian, W. Jiang, Y. Gao, and F. Fu, "Therapeutic effects of SMND-309, a new metabolite of salvianolic acid B, on experimental liver fibrosis," European Journal of Pharmacology, vol. 650, no. 1, pp. 390-395, 2011.

[25] S. Hara, T. Mukai, K. Kurosaki, F. Kuriiwa, and T. Endo, "Characterization of suppression of nitric oxide production by carbon monoxide poisoning in the striatum of free-moving rats, as determined by in vivo brain microdialysis," Brain Research, vol. 979, no. 1-2, pp. 27-36, 2003.

[26] S. Hara, T. Mukai, K. Kurosaki, H. Mizukami, F. Kuriiwa, and T. Endo, "Role of nitric oxide system in hydroxyl radical generation in rat striatum due to carbon monoxide poisoning, as determined by microdialysis," Toxicology, vol. 239, no. 1-2, pp. 136-143, 2007.

[27] M.-K. Tang, D.-C. Ren, J.-T. Zhang, and G.-H. Du, "Effect of salvianolic acids from Radix Salviae miltiorrhizae on regional cerebral blood flow and platelet aggregation in rats," Phytomedicine, vol. 9, no. 5, pp. 405-409, 2002.

[28] H.-K. Zeng, Q.-S. Wang, Y.-Y. Deng et al., "A comparative study on the efficacy of $10 \%$ hypertonic saline and equal volume of $20 \%$ mannitol in the treatment of experimentally induced cerebral edema in adult rats," BMC Neuroscience, vol. 11, article 153,2010

[29] W. Yao, Z. Wen, Z. Yan et al., "Low viscosity Ektacytometry and its validation tested by flow chamber," Journal of Biomechanics, vol. 34, no. 11, pp. 1501-1509, 2001.

[30] N. Mohandas and J. A. Chasis, "Red blood cell deformability, membrane material properties and shape: regulation by transmembrane, skeletal and cytosolic proteins and lipids," Seminars in Hematology, vol. 30, no. 3, pp. 171-192, 1993.

[31] J. Zhang, "Effect of suspending viscosity on red blood cell dynamics and blood flows in microvessels," Microcirculation, vol. 18, no. 7, pp. 562-573, 2011.

[32] Z. Y. Wen, L. C. Song, Z. Y. Yan et al., "An animal model to study erythrocyte senescence with a narrow time window of erythrocyte production: alterations in osmotic fragility and deformability of erythrocytes during their life span," Clinical Hemorheology and Microcirculation, vol. 19, no. 4, pp. 299-306, 1998. 
[33] G. Cicco and A. Pirrelli, "Red blood cell (RBC) deformability, RBC aggregability and tissue oxygenation in hypertension," Clinical Hemorheology and Microcirculation, vol. 21, no. 3-4, pp. 169-177, 1999.

[34] M. Condon, M. Senthil, D.-Z. Xu et al., "Intravenous injection of mesenteric lymph produced during hemorrhagic shock decreases RBC deformability in the rat," The Journal of Trauma, vol. 70, no. 2, pp. 489-495, 2011.

[35] G. Barshtein, N. Manny, and S. Yedgar, "Circulatory risk in the transfusion of red blood cells with impaired flow properties induced by storage," Transfusion Medicine Reviews, vol. 25, no. 1, pp. 24-35, 2011.

[36] G. Barshtein, R. Ben-Ami, and S. Yedgar, "Role of red blood cell flow behavior in hemodynamics and hemostasis," Expert Review of Cardiovascular Therapy, vol. 5, no. 4, pp. 743-752, 2007.

[37] G. B. Nash, T. Watts, C. Thornton, and M. Barigou, "Red cell aggregation as a factor influencing margination and adhesion of leukocytes and platelets," Clinical Hemorheology and Microcirculation, vol. 39, no. 1-4, pp. 303-310, 2008.

[38] O. K. Baskurt, "In vivo correlates of altered blood rheology," Biorheology, vol. 45, no. 6, pp. 629-638, 2008.

[39] K. R. Hardy and S. R. Thom, "Pathophysiology and treatment of carbon monoxide poisoning," Journal of Toxicology-Clinical Toxicology, vol. 32, no. 6, pp. 613-629, 1994.

[40] X. Wang, T. Wen, L. Guan, Y. Zhang, M. Zhu, and J. Zhao, "Hemorheological changes in cerebral circulation of rabbits with acute carbon monoxide poisoning," Clinical Hemorheology and Microcirculation, vol. 43, no. 4, pp. 271-282, 2009. 

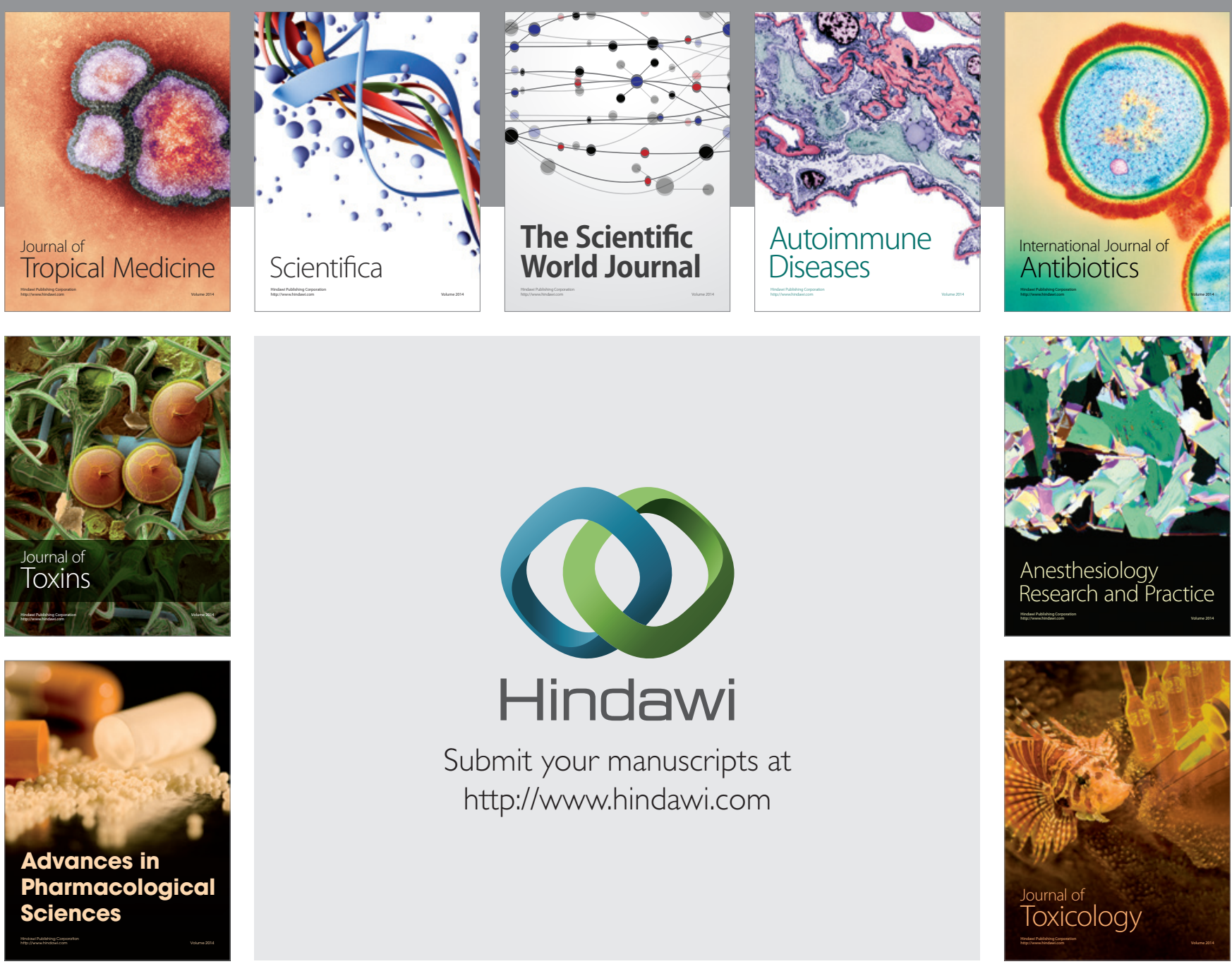

\section{Hindawi}

Submit your manuscripts at

http://www.hindawi.com
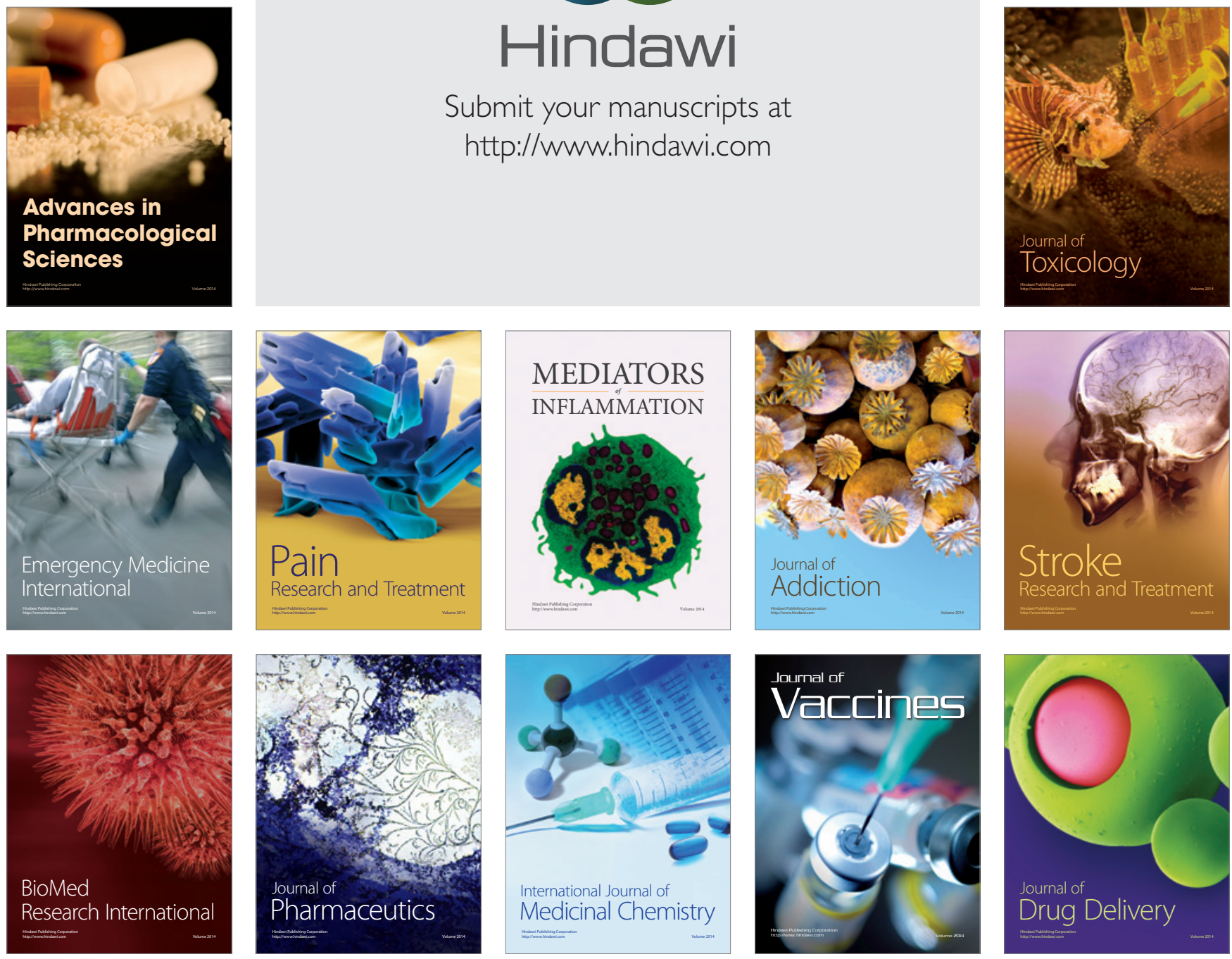